\title{
WI - Call for Papers Heft 3/2012
}

\section{Informationssysteme und kulturelle Einflussfaktoren}

DOI 10.1007/s11576-010-0250-2

\section{Die Autoren \\ Prof. Dr. Armin Heinzl ( $\varangle)$ \\ Universität Mannheim \\ Schloss \\ 68131 Mannheim \\ Deutschland \\ heinzl@uni-mannheim.de}

Prof. Dr. Dorothy E. Leidner

Hankamer School of Business

Baylor University

P.O. Box 98005

Waco, TX 76798-8005,

USA

Dorothy_Leidner@baylor.edu

Online publiziert: 2010-10-15

This article is also available in English via http://www.springerlink.com and http://www.bise-journal.org: Heinzl A, Leidner DE (2010) BISE - Call for Papers Issue 3/2012. Information Systems and Culture. Bus Inf Syst Eng. doi: 10.1007/s12599-010-0133-5.

(c) Gabler Verlag 2010

\section{Schwerpunktthema}

Informationssysteme (IS) sind soziotechnische Gebilde, in denen bestimmte Aufgaben durch Menschen mithilfe von Informations- und Kommunikationstechnologien erfüllt werden. Der Erfolg der Entwicklung, Einführung und Nutzung von Informationssystemen hängt $u$. a. stark vom kulturellen Kontext ab, in den sie eingebettet sind. Kultur bezieht sich dabei auf die Einstellungen, Werte, Ziele und Bräuche beteiligter Aufgabenträger auf organisationaler, beruflicher und nationaler Ebene. Kulturelles Bewusstsein ist ein Schlüssel zur Vermeidung von Problemen und zur Sicherstellung einer bestmöglichen Kongruenz zwischen Technologie, Aufgabe und Mensch.
In den vergangenen Jahren haben Aufgabenträger unterschiedlicher Berufsgruppen aus Ländern mit unterschiedlichem kulturellem Hintergrund ihre Kooperationsbestrebungen erhöht, um neue Informationssysteme und systemnahe Dienstleistungen erfolgreich zu entwickeln und zu betreiben. Der Erfolg von Nearshoring- und Offshoring-Projekten hängt maßgeblich davon $\mathrm{ab}$, wie kulturelle Unterschiede auf der Anbieterund Kundenseite überbrückt werden. Darüber hinaus haben international agierende IT-Dienstleister globale Liefermodelle (global delivery models) entwickelt, die versuchen, lokale und entfernte Standorte $\mathrm{zu}$ vernetzen, um spezifische Kulturvorteile an den jeweiligen Standorten auszunutzen. Im Bereich der Unternehmenssoftware können die kulturellen Praktiken und Annahmen auf der Anbieterseite stark von denen auf der Kundenseite abweichen. Ein Kundenunternehmen in Brasilien folgt beispielsweise kulturell bedingt anderen Denk- und Handlungsmustern in Geschäftsprozessen als ein ERP-Anbieter aus Deutschland. Während der Systemnutzung müssen Informationssysteme die kulturellen Hintergründe unterschiedlicher Berufsgruppen sowie die Unternehmensgrenzen überwinden. Auch hier stellt die Berücksichtigung und Bewältigung kultureller Faktoren ein Schlüsselmerkmal des Systemerfolgs dar.

Dieses Schwerpunktheft der Zeitschrift WIRTSCHAFTINFORMATIK verfolgt das Ziel, neue wissenschaftliche Erkenntnisse sowie Erfahrungen aus Theorie und Praxis zu behandeln, die kulturelle Aspekte in jeder Phase des ISLebenszyklus in den Mittelpunkt ihrer Überlegungen stellen.

Beiträge aus Forschung und Praxis sind u. a. zu folgenden (gerne auch weiteren) Themenfeldern erwünscht:

- Nationale und organisatorische Fragen zu kulturellen Aspekten in Informationssystemen

- Theorien und Modelle, die sich mit kulturellen Fragen in Informationssystemen beschäftigen
- Der Einfluss kultureller Faktoren auf die Wahl von Forschungsmethoden

- Forschungsmethoden, die spezifisch für Kulturforschung in Informationssystemen geeignet sind

- Kulturvergleichende Studien im Zusammenhang mit Informationssystemen und Informationstechnologien

- Kultur und Eigenschaften der Benutzer von Informationssystemen (z. B. Entwickler, Anwender, Berater, etc.)

- Beschreibungs-, Mess- und Erklärungskonzepte für kulturelle Faktoren in Informationssystemen

- Fallstudien, die sich mit der Entwicklung, Einführung, Adoption und Verbreitung von Informationssystemen in unterschiedlichen Kulturräumen beschäftigen

- Die Rolle des Geschlechts im Rahmen kultureller Aspekte bei der Entwicklung und Nutzung von Informationssystemen

- Konzepte und Beispiele kultureller Intelligenz

- Weitere Fragestellungen im Zusammenhang mit kulturellen Aspekten im Zusammenhang mit Informationssystemen

\section{Einreichung von Beiträgen}

Bitte reichen Sie Beiträge für die Rubriken WI - Aufsatz und WI - State of the Art bis spätestens 2011-07-01 über das Online-Begutachtungssystem (http:// www.editorialmanager.com/buis/) ein. Bitte beachten Sie die Hinweise zu formaler Gestaltung und Umfang von Beiträgen für die WIRTSCHAFTSINFORMATIK/Business \& Information Systems Engineering (BISE). Vollständige Beiträge sollten höchstens zehn Druckseiten umfassen; das entspricht 50.000 Zeichen einschließlich Leerzeichen, abzüglich 5.000 Zeichen je Seite an Bildern. Ausführliche Autorenrichtlinien stehen unter http://www. wirtschaftsinformatik.de zum Download bereit. 
Eingereichte Beiträge werden anonymisiert von mehreren Gutachtern in einem doppelt-blinden Verfahren auf Relevanz, Originalität und fachliche Qualität beurteilt. Neben den Herausgebern des Schwerpunktthemas und jenen der Zeitschrift wirken dabei weitere ausgewiesene internationale Persönlichkeiten aus Wissenschaft und Praxis mit.

Ergänzend zu den Aufsätzen sind auch weitere Einreichungen zum Schwerpunktthema willkommen.
Angenommene Beiträge erscheinen identisch in Deutsch und Englisch. Die deutschsprachige Fassung erscheint in WIRTSCHAFTSINFORMATIK, die englischsprachige in Business \& Information Systems Engineering (BISE). Angenommene Beiträge werden in enger Zusammenarbeit von Autoren und einem professionellen Übersetzerteam übersetzt.

\section{Zeitplan}

Einreichung von Beiträgen: 2011-07-01
Benachrichtigung der Autoren: 2011-08-26

Abschluss der ersten Überarbeitung: 2011-10-28

Benachrichtigung der Autoren:

2011-12-16

Ggf. Abschluss einer zweiten Überarbeitung (einsprachig): 2012-01-20

Ggf. Abschluss einer zweiten Überarbeitung (zweisprachig): 2012-02-17 Geplanter Erscheinungstermin Heft 3/2012: Juni 2012 